\title{
Zero-Point Fluctuations and the Quenching of the Persistent Current in Normal Metal Rings
}

\author{
Pascal Cedraschi, Vadim V. Ponomarenko, and Markus Büttiker \\ Département de Physique Théorique, Université de Genève, \\ 24, quai Ernest Ansermet, CH-1211 Geneva 4, Switzerland
}

(August 30, 2018)

\begin{abstract}
The ground state of a phase-coherent mesoscopic system is sensitive to its environment. We investigate the persistent current of a ring with a quantum dot which is capacitively coupled to an external circuit with a dissipative impedance. At zero temperature, zero-point quantum fluctuations lead to a strong suppression of the persistent current with decreasing external impedance. We emphasize the role of displacement currents in the dynamical fluctuations of the persistent current and show that with decreasing external impedance the fluctuations exceed the average persistent current.
\end{abstract}

PACS numbers: 73.23.Ra, 73.23.Hk, 71.27.+a

The persistent current in a doubly connected conductor penetrated by an Aharonov-Bohm flux is an indicator of quantum coherent electron motion in the ground state of the system. Only the charge carriers whose wave functions are sufficiently extended to reach around the loop can carry a persistent current [1]. In this letter, we are interested in the effect of zero-point fluctuations of an environment on the ground state of the system. We consider a mesoscopic ring with an in-line quantum dot, coupled capacitively to a polarizable, dissipative environment, modeled by an impedance $Z$, see Fig. 1. The fluctuations leading to exchange of charge between the dot and the arm of the ring are capacitively coupled to the charge fluctuations of the external circuit. We investigate the zero-temperature limit of this system in which the zero-point fluctuations of the loop interact with the zero-point fluctuations of the external circuit. We show that with decreasing external impedance the persistent current in this system is strongly suppressed below its value for infinite impedance. Thus the zero-temperature environment can effectively destroy the coherence of the mesoscopic system. The role of zero-temperature fluctuations and its effect on phase-coherent transport properties is a subject of high current interest [2]. While the coherence properties of the ground state for superconducting structures has been discussed [3], a discussion for normal systems seems not to be available.

We investigate the properties of the ground state of the structure depicted schematically in Fig. 1 with particular emphasis on the influence of Coulomb interactions on the currents. The Coulomb interactions will be treated in a capacitive model which assumes that well defined regions can be described with a single potential. In the conductor of Fig. 1 the regions are the $\operatorname{dot}\left(U_{d}\right)$, the arm $\left(U_{a}\right)$ and the external circuit regions $\left(V_{0}, V_{\infty}\right)$ separated by capacitors $C_{1}$ and $C_{2}$ from the ring. Our first task is to derive expressions for the currents valid in the presence of potential fluctuations. For the dynamical fluctuations

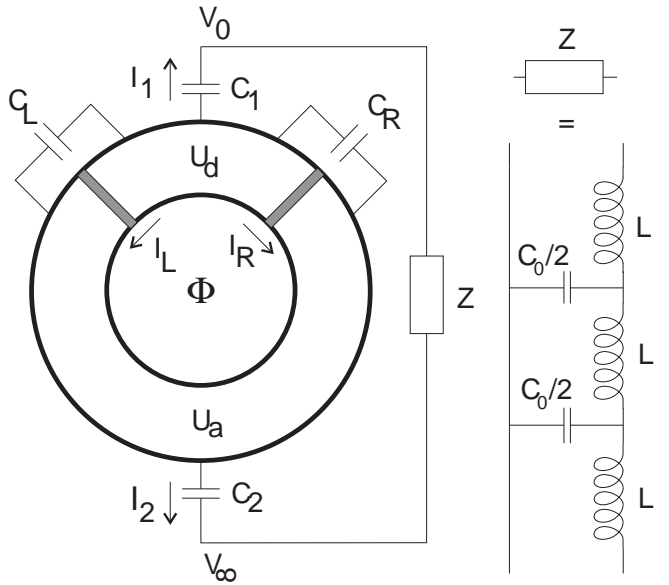

FIG. 1. Ring with an in-line dot subject to a flux $\Phi$ and capacitively coupled to an external impedance $Z$.

of interest here it is important to take into account that only total currents, namely particle currents augmented by displacement currents, are conserved quantities. It is the total currents which are related to the magnetization and its fluctuations. In terms of the particle currents $I_{L}^{p}$ and $I_{R}^{p}$ through the left and right tunnel barrier characterized by capacitances $C_{L}$ and $C_{R}$ the total currents are

$$
\begin{aligned}
I_{L} & =I_{L}^{p}+C_{L} \frac{\partial}{\partial t}\left(U_{d}-U_{a}\right), \\
I_{R} & =I_{R}^{p}+C_{R} \frac{\partial}{\partial t}\left(U_{d}-U_{a}\right), \\
I_{1} & =C_{1} \frac{\partial}{\partial t}\left(U_{d}-V_{0}\right) .
\end{aligned}
$$

In $I_{1}$ the potential $V_{0}$ characterizes the external circuit. Eqs. (1-3) are quantized by replacing $I_{L}^{p}$ and $I_{R}^{p}$ by the corresponding particle current operators $\hat{I}_{L, R}^{p}$ and by replacing time derivatives by commutators with the Hamiltonian of the entire system $\partial A / \partial t \rightarrow i / \hbar[\hat{H}, \hat{A}]$. Note 
that these currents satisfy Kirchhoff's rule $I_{L}+I_{R}+I_{1}=$ 0 , and thus are conserved, even when quantized. In the ground state the average currents through the left and right junctions are equal to each other and both equal (in magnitude) to the persistent current, $\left\langle\hat{I}_{L}\right\rangle=-\left\langle\hat{I}_{R}\right\rangle=$ $I=-c \partial F / \partial \Phi$, where $F$ is the free energy. However, the dynamical currents, in particular, the fluctuations of $\hat{I}_{L}$ and $\hat{I}_{R}$ are usually different even in the ground state and depend explicitly on the capacitances.

The circuit of Fig. 1 consists of two loops and can thus be characterized by the displacement current through the external loop, $\hat{I}_{1}=-\left(\hat{I}_{L}+\hat{I}_{R}\right)=-\hat{I}_{2}$ and a current circulating around the ring with the quantum dot, $\hat{I}_{c}$. Taking into account that the displacement current $\hat{I}_{1}$ is divided over the two branches of the circuit according to the capacitances $C_{R}$ and $C_{L}$, we find the circulating current

$$
\hat{I}_{c}=\frac{C_{R} \hat{I}_{L}-C_{L} \hat{I}_{R}}{C}
$$

with $C \equiv C_{L}+C_{R}$. Note that according to Eqs. (1) and (2) this current depends on the capacitances and the particle portion of the total current. Such an expression for the current, Eq. (雨), is familiar from dynamical investigation of resonant tunneling in double barrier structures, where it is known as Ramo-Shockly theorem, but seems novel in the context of persistent currents.

We model the finite impedance $Z$ in a Hamiltonian approach, with the help of a transmission line, see Fig. 1, with capacitance $C_{0}$ and inductance $L$. For convenience we take the capacitance to coincide with the effective capacitance of the mesoscopic structure, $C_{0}^{-1} \equiv C^{-1}+C_{e}^{-1}$ with $C_{e}^{-1} \equiv C_{1}^{-1}+C_{2}^{-1}$. The ohmic resistance generated by the transmission line is $R \equiv Z(\omega=0)=\left(2 L / C_{0}\right)^{1 / 2}$ and thus can be varied by adjusting the inductance. There are other ways to model a resistance, and general rules are given in Ref. [4]. We denote the charges on the capacitors between the inductances by $Q_{n}$ and the potentials by $V_{n}, n=0,1,2, \ldots$. Furthermore, we introduce the generalized fluxes $\phi_{n}$ satisfying the equations $d \phi_{n} / d t=V_{n}-V_{\infty}$. The charge on the dot is $Q_{d}$, and the charge on the capacitor $C_{1}$ is $Q_{0}$. Then the Hamiltonian including all electromagnetic interactions reads

$$
\begin{aligned}
H_{C} & =\frac{Q_{d}^{2}}{2 C}+\frac{Q_{d} Q_{0}}{C}+\frac{Q_{0}^{2}}{2 C_{0}} \\
& +\sum_{n=1}^{\infty}\left\{\frac{Q_{n}^{2}}{C_{0}}+\frac{\left(\phi_{n}-\phi_{n-1}\right)^{2}}{2 L}\right\} .
\end{aligned}
$$

Eq. (5) is quantized by replacing the charge and the generalized flux by operators obeying the commutation relations $\left[\hat{\phi}_{m}, \hat{Q}_{n}\right]=i \hbar \delta_{m n}$. The infinite sum in Eq. (5) is diagonalized by the transformation $Q_{n}=$ $\int_{0}^{1} d x\left[Q(x)+\left(C_{0} / C\right) e\left(N_{+}+1 / 2\right)\right] \exp (-2 \pi i n x), \phi_{n}=$ $\int_{0}^{1} d x \phi(x) \exp (-2 \pi i n x)$, giving rise to a bath of uncoupled harmonic oscillators

$$
\hat{H}_{H O}=\int_{0}^{1} d x\left\{\frac{\sin ^{2} \pi x}{L} \hat{\phi}^{2}(x)+\frac{\hat{Q}^{2}(x)}{2 C_{0}}\right\}
$$

We observe that the spectrum of $\hat{H}_{H O}$ is dense, such that there are no recurrence phenomena [5. This electromagnetic Hamiltonian, Eq. (5), has to be augmented by the Hamiltonian of the electronic degrees of freedom in the ring. We consider only spinless electrons in this letter and describe the coupling of the dot and arm in the limit in which the tunneling amplitudes through the left and the right junction are much smaller in magnitude than the level spacing in the dot and the level spacing in the arm. In this limit, discussed by Stafford and one of the authors [6], one may consider hybridization between the topmost occupied electron level in the arm and the lowest unoccupied level in the dot, $\epsilon_{a M}$ and $\epsilon_{d(N+1)}$ only. We denote the tunneling amplitudes between the levels $\epsilon_{a M}$ and $\epsilon_{d(N+1)}$ by $t_{L}$ for tunneling through the left junction and by $t_{R}$ for tunneling through the right one. The total tunneling amplitude between the dot and the arm, is a function of the Aharonov-Bohm flux $\Phi$ through the ring and is given by $\hbar \Delta_{0} / 2 \equiv\left(t_{L}^{2}+t_{R}^{2} \pm 2 t_{L} t_{R} \cos \varphi\right)^{1 / 2}$. The sign is positive if the number of electrons in the ring is odd, and negative otherwise, and $\varphi \equiv 2 \pi \Phi / \Phi_{0}$ is the AharonovBohm flux in units of the flux quantum $\Phi_{0}=h c / e$. Now, the Hamiltonian of the electronic degrees of freedom reads $\hat{H}_{e}=\left[\left(\epsilon_{a M}-\epsilon_{d(N+1)}\right) / 2\right] \sigma_{z}-\left(\hbar \Delta_{0} / 2\right) \sigma_{x}$, and the charge on the dot is $\hat{Q}_{d}=(e / 2) \sigma_{z}+e\left(N-N_{+}+1 / 2\right)$, where $\sigma_{z}$ and $\sigma_{x}$ are Pauli matrices and $N_{+}$is the number of background charges on the dot. Due to the interactions, Eq. ([5), the detuning $\epsilon_{a M}-\epsilon_{d(N+1)}$ is changed into $\hbar \varepsilon \equiv \epsilon_{a M}-\epsilon_{d(N+1)}+\left(e^{2} / C\right)\left(N-N_{+}+1 / 2\right)$. The tunneling described by the term in $\sigma_{x}$ is dressed by the interactions with the dissipative circuit. The complete Hamiltonian becomes

$$
\hat{H}=\frac{\hbar \varepsilon}{2} \sigma_{z}+\hat{H}_{H O}-\frac{\hbar \Delta_{0}}{2}\left(\sigma_{+} e^{-i \hat{\Omega}}+\sigma_{-} e^{i \hat{\Omega}}\right),
$$

where $\sigma_{ \pm}=1 / 2\left(\sigma_{x} \pm i \sigma_{y}\right)$ and $\hat{\Omega}=(e / \hbar)\left(C_{0} / C\right) \hat{\phi}_{0}$. Eq. (7) is the Caldeira-Leggett (CL) model [5:]]. To make the connection to this model more explicit, we introduce the RC-time $\tau_{R C}=R C$ and the parameters $\omega_{c}=\pi \omega_{0} / 2$ and the coupling strength

$$
\alpha \equiv \frac{\pi}{2} \frac{e^{2} /(2 C)}{\hbar \omega_{c}} \frac{1}{\omega_{c} \tau_{R C}} .
$$

These parameters also determine the spectral density $J(\omega)=2 \pi \hbar \alpha \omega \exp \left(-\omega / \omega_{c}\right)$. The coupling between the ring and the external circuit is mediated by the operator $\hat{\Omega}$. We shall therefore investigate its correlator, $\left\langle e^{-i \hat{\Omega}(t)} e^{i \hat{\Omega}(0)}\right\rangle$, where $\hat{\Omega}(t) \equiv e^{-i \hat{H} t / \hbar \hat{\Omega}} e^{i \hat{H} t / \hbar}$, in particular its long time behavior. In imaginary time, $\tau=i t$, it reads 


$$
\begin{aligned}
\left\langle e^{-i \hat{\Omega}(\tau)} e^{i \hat{\Omega}(0)}\right\rangle & =\exp \left[-\int_{0}^{\infty} d \omega \frac{J(\omega)}{\omega^{2}}\left(1-e^{-\omega|\tau|}\right)\right] \\
& =\left(1+\omega_{c}|\tau|\right)^{-2 \alpha} .
\end{aligned}
$$

We note that $\alpha$ is proportional to the Coulomb energy $e^{2} /(2 C)$, and inversely proportional to the resistance $R$. In the next section we discuss the ground state of Eq. (7) for different values of the coupling strength $\alpha$.

For $\alpha=0$, the ring is not coupled to the bath of harmonic oscillators, and the ground state is obtained by diagonalizing the $2 \times 2$-matrix $\hat{H}_{\alpha=0}=$ $(\hbar \varepsilon / 2) \sigma_{z}-\left(\hbar \Delta_{0} / 2\right) \sigma_{x}$. The persistent current is $I=e / 2\left(\varepsilon^{2}+\Delta_{0}^{2}\right)^{-1 / 2} \Delta_{0}\left(\partial \Delta_{0} / \partial \varphi\right)$ with $\hbar\left(\partial \Delta_{0} / \partial \varphi\right)=$ $\left(\mp t_{L} t_{R} \sin \varphi\right) / \sqrt{t_{L}^{2}+t_{R}^{2} \pm 2 t_{l} t_{R} \cos \varphi}$ [6]. It is maximal at resonance, $\varepsilon=0$, where it takes the value $(e / 2) \partial \Delta_{0} / \partial \varphi$, and decreases like $\varepsilon^{-1}$ far away from resonance. The persistent current is not only equal to the average of the circulating current, $I=\left\langle\hat{I}_{c}\right\rangle$, but also equal (in magnitude) to the total as well as to the particle currents through the left and the right junctions, $I=\left\langle\hat{I}_{L}\right\rangle=\left\langle\hat{I}_{L}^{p}\right\rangle=-\left\langle\hat{I}_{R}\right\rangle=-\left\langle\hat{I}_{R}^{p}\right\rangle$. The current through the ring vanishes on the average, $\left\langle\hat{I}_{1}\right\rangle=0$. Thus the average currents are pure particle currents.

In the fluctuations of the currents, however, displacement currents play an important role. In our model with only two relevant electron levels, the fluctuations of the circulating current are given by the general formula

$$
\begin{aligned}
\Delta I_{c}{ }^{2} & =\left(\frac{e}{2} \frac{\partial \Delta_{0}}{\partial \varphi}\right)^{2}-I^{2} \\
& +\frac{e^{2}}{\hbar^{2}} \frac{\left[C_{R} t_{L}^{2}-C_{L} t_{R}^{2} \pm\left(C_{R}-C_{L}\right) t_{L} t_{R} \cos \varphi\right]^{2}}{C^{2}\left(t_{L}^{2}+t_{R}^{2} \pm 2 t_{L} t_{R} \cos \varphi\right)} .
\end{aligned}
$$

The first two terms are due to the particle currents through the junctions whereas the last one is of electromagnetic origin. Eq. (10) holds also when the ring is coupled to the environment. We point out that the fluctuations of the current are minimal at resonance, where the current itself is maximal. Note that only the persistent current $I$ is changed by interaction with the environment. This is a consequence of the effective two level description of our system. The fluctuations of the current through the ring, $\hat{I}_{1}=(2 / L) \int_{0}^{1} d x \sin ^{2} \pi x \hat{\phi}(x)$ are entirely due to the displacement current, and for $\alpha=0$, they read

$$
\Delta I_{1}^{2}=\frac{8}{3 \pi} e^{2} \frac{\hbar \omega_{c}}{e^{2} /(2 C)} \frac{\omega_{c}}{\tau_{R C}}
$$

They are inversely proportional to the charging energy as well as to the resistance, thus they decrease with increasing charging energy, and increase with increasing dissipation (decreasing $R$ ). For $\alpha \neq 0$, and far from resonance (even at resonance for $\alpha>1$ ), these fluctuations receive perturbative corrections in $\Delta_{0} / \omega_{c}$. The dominating contribution to $\Delta I_{1}^{2}$, however, comes from the high frequency oscillators, and thus $\Delta I_{1}{ }^{2}$ is not model independent.

We shall now determine the properties of the ground state at arbitrary $\alpha>0$. It is useful to mention at this point that the CL Hamiltonian, Eq. (『), may be mapped [5] on the Hamiltonian of the anisotropic Kondo model as well as on the Hamiltonian of the resonant level model. For a vanishing magnetic field, corresponding to our model being at resonance, $\varepsilon=0$, the Kondo model is characterized by two parameters, which may be identified as $\alpha$ and $\Delta_{0} / \omega_{c}$. The half plane spanned by $\Delta_{0} / \omega_{c} \geq 0$ and $\alpha$ is divided up into three regions by the separatrix equation $|1-\alpha|=\Delta_{0} / \omega_{c}$, where the parameters flow to different fixed points under the action of the renormalization group [8]. We are not interested in the regime of strong tunneling $\Delta_{0} / \omega_{c}>|1-\alpha|$. Of the remaining two, the region $\alpha>1, \Delta_{0} / \omega_{c}<\alpha-1$ corresponds to the ferromagnetic Kondo model where $\Delta_{0} / \omega_{c}$ renormalizes to zero, the region $\alpha<1, \Delta_{0} / \omega_{c}<1-\alpha$ corresponds to the anti-ferromagnetic Kondo model that has been solved by Bethe ansatz [9]. For large detuning $\varepsilon$, both cases can be treated by a perturbative expansion in $\Delta_{0}$.

At zero temperature, to second order in $\Delta_{0}$, the free energy is $F_{0}+\delta F$ with $F_{0}=-\hbar|\varepsilon| / 2$ and $\delta F=$

$$
-\frac{\hbar \omega_{c}}{4}\left(\frac{\Delta_{0}}{\omega_{c}}\right)^{2} e^{|\varepsilon| / \omega_{c}}\left|\frac{\varepsilon}{\omega_{c}}\right|^{2 \alpha-1} \Gamma\left(1-2 \alpha, \frac{|\varepsilon|}{\omega_{c}}\right)
$$

where $\Gamma(\zeta, x)$ is the incomplete gamma function. Eq. (12) is valid at any $\alpha$ up to second order in $\Delta_{0} / \omega_{c}$ and for large detuning $\varepsilon$. In the regime $\alpha-1 \gg \Delta_{0} / \omega_{c}$ (the ferromagnetic regime of the Kondo model), Eq. (12) is valid even for arbitrary detuning $\varepsilon$. It is well known [10] that the CL model exhibits symmetry breaking for $\alpha>1$, namely $\left\langle\sigma_{z}\right\rangle$ exhibits a finite jump as $\varepsilon$ crosses zero. In perturbation theory the width of this jump is $\left\langle\sigma_{z}\right\rangle_{\varepsilon=0-}-\left\langle\sigma_{z}\right\rangle_{\varepsilon=0+}=1-\left(\Delta_{0} / \omega_{c}\right)^{2} /(2(2 \alpha-1)(2 \alpha-2))$. In the persistent current, it shows up as a cusp at resonance.

For $0<\alpha<1$, we use the Bethe ansatz solution of the anti-ferromagnetic Kondo model, or rather of the equivalent resonant level model [11. The low energy properties of the problem depend on three energy scales, namely the detuning $\varepsilon$, a cutoff $D$ and the "Kondo temperature" $T_{K}=\Delta_{0}\left(\Delta_{0} / D\right)^{(\alpha /(1-\alpha))}$, which is the only scale that depends on the magnetic flux $\varphi$. The persistent current is calculated from the known expression [1] for $\left\langle\sigma_{z}\right\rangle=\partial F / \partial(\hbar \varepsilon)$

$$
\begin{aligned}
\left\langle\sigma_{z}\right\rangle=\frac{i}{4 \pi^{3 / 2}} & \int_{-\infty}^{\infty} \frac{d p}{p-i 0} e^{i p(\ln z+b)} \\
& \times \frac{\Gamma(1+i p) \Gamma(1 / 2-i(1-\alpha) p)}{\Gamma(1+i \alpha p)}
\end{aligned}
$$




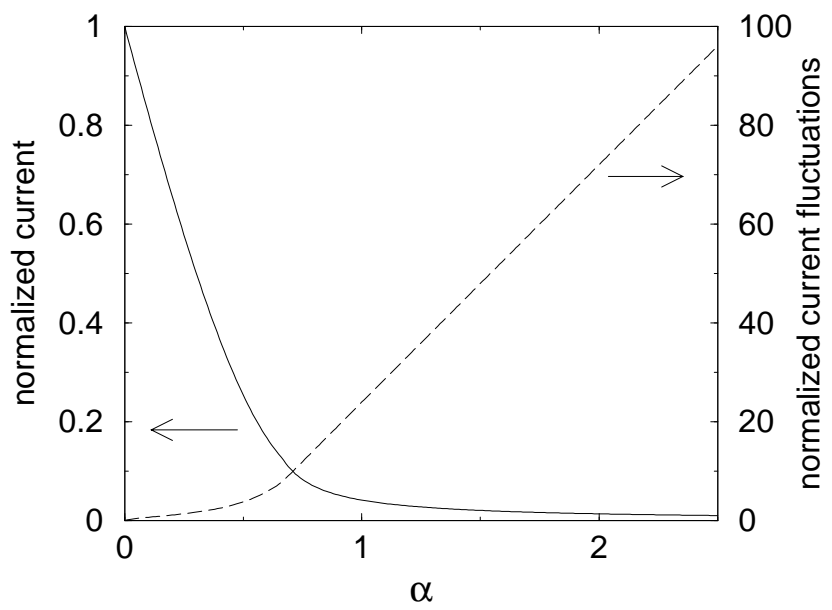

FIG. 2. The persistent current at resonance (solid line) in units of the current at $\alpha=0$ and the normalized fluctuations of the circulating current $\sqrt{\Delta I_{c}^{2}} / I$ (dashed line). The parameters are $\omega_{c}=25 \Delta_{0}$.

where $z=\left(\varepsilon / T_{K}\right)^{2(1-\alpha)}$ and $b=\alpha \ln \alpha+(1-\alpha) \ln (1-\alpha)$. This Bethe ansatz solution is valid only for $1-\alpha \gg$ $\Delta_{0} / \omega_{c}$. Now, we make use of the fact that in terms of the above-mentioned energy scales, the free energy reads $F=\hbar T_{K} f\left(\varepsilon / T_{K}, T_{K} / D\right)$, and thus the persistent current $I=-(e / \hbar)(\partial F / \partial \varphi)$ may be expressed in terms of $\left\langle\sigma_{z}\right\rangle=\partial F / \partial(\hbar \varepsilon)$

$$
I=-e \frac{\partial T_{K}}{\partial \varphi}\left[\int_{0}^{y} d x\left\langle\sigma_{z}\right\rangle(x)-y\left\langle\sigma_{z}\right\rangle(y)\right]+I_{0} .
$$

where $y \equiv \frac{\varepsilon}{T_{K}}$. We point out that $T_{K}$ sets the scale for the transition from resonant to perturbative behavior, i.e. for $\varepsilon \gg T_{K}$, the expressions for the persistent current from Bethe ansatz and from the perturbation theory must coincide up to terms of order $\Delta_{0}$. This observation allows us to determine the cutoff $D$ in terms of $\omega_{c}$,

$$
\left(\frac{D}{\omega_{c}}\right)^{2 \alpha}=\frac{2 \Gamma(3 / 2-\alpha) e^{-b}}{\sqrt{\pi}(1-2 \alpha) \Gamma(1-2 \alpha) \Gamma(1-\alpha)},
$$

as well as the integration constant $I_{0}$.

$$
\begin{aligned}
I_{0}= & -\frac{e}{2} \frac{\Gamma\left(1-\frac{1}{2(1-\alpha)}\right) e^{-\frac{b}{2(1-\alpha)}}}{\sqrt{\pi}(1-\alpha) \Gamma\left(1-\frac{\alpha}{2(1-\alpha)}\right)}\left(\frac{\Delta_{0}}{D}\right)^{\frac{\alpha}{1-\alpha}} \frac{\partial \Delta_{0}}{\partial \varphi} \\
& +\frac{e}{2} \frac{1}{1-2 \alpha} \frac{\Delta_{0}}{\omega_{c}} \frac{\partial \Delta_{0}}{\partial \varphi} .
\end{aligned}
$$

The poles in the first term in Eq. (16) appearing at $\alpha=(2 n+1) /(2 n+2)$ for $n \geq 0$ are canceled by terms of higher order in $\Delta_{0}$. For $\alpha<1 / 2$, the first term in Eq. (16), behaving like $\left(\Delta_{0} / D\right)^{\alpha /(1-\alpha)}$ is dominating. For $\alpha>1 / 2$ the second one dominates and behaves as $\Delta_{0} / D$. The power law for $\alpha>1 / 2$ is thus the same as one obtains from perturbation theory at $\alpha>1$, using $I=-(e / \hbar)(\partial F / \partial \varphi)$ with $F$ given by Eq. (12). It is known that the lowest excited state [5] loses its phase coherence at $\alpha=1 / 2$. However, the persistent current remains differentiable in $\varepsilon$ as $\alpha$ passes through $1 / 2$ and the ground state retains some coherence even for large $\alpha$. As a function of detuning the cusp at resonance shows up only at $\alpha>1$. The poles at $\alpha=1 / 2$ in the two terms in Eq. (16) cancel each other. Together they give rise to a logarithmic persistent current

$$
I_{0}\left(\alpha=\frac{1}{2}\right) \approx e \frac{\partial \Delta_{0}}{\partial \varphi} \frac{\Delta_{0}}{\omega_{c}}\left(\ln \frac{\Delta_{0}}{\omega_{c}}-0.217 \ldots\right) .
$$

The logarithmic term $\ln \left(\Delta_{0} / \omega_{c}\right)$ characterizes the transition from power law to linear behavior. The average circulating current, Eq. (14), is shown in Fig. 2 as function of $\alpha$ together with the mean squared fluctuations of the circulating current obtained by using Eqs. (14, 16) and Eq. (10).

Let us discuss some orders of magnitude. Assume that the bosonic excitations described by the bath of harmonic oscillators, Eq. (6), are surface plasmons. Thus $\omega_{c}$ is approximately the frequency of a surface plasmon $\omega_{p}$, which is of the order of $\omega_{p} \approx 10^{14} s^{-1}$. If we a use a typical mesoscopic capacitance of $C \approx 10^{-16} F$, and $R \approx 1 \Omega$, we obtain $\alpha \approx 1(!)$. Clearly a large range of coupling constants can be experimentally realized and thus permit the investigation of the effect of the zero-point fluctuations on the persistent current, and thus on the ground state, of a mesoscopic system.

This work was supported by the Swiss National Science Foundation.

[1] M. Büttiker, Y. Imry, and R. Landauer, Phys. Lett. 96A, 365 (1983).

[2] P. Mohanty, E. M. Q. Jariwala, and R. A. Webb, Phys. Rev. Lett. 78, 3366 (1997); I. L. Aleiner, B. L. Altshuler, and M. E. Gershenson, ibid 82, 3190 (1999); D. S. Golubev and A. D. Zaikin, ibid 82, 3191 (1999).

[3] F. W. J. Hekking and L. I. Glazman, Phys. Rev. B55, 6551 (1997). M. Oshikawa and A. M. Zagoskin, condmat/9810303.

[4] G.-L. Ingold and Y. V. Nazarov, in Single Charge Tunneling: Columb Blockade Phenomena in Nanostructures, Vol. 294 of NATO ASI series, edited by H. Grabert and M. H. Devoret (Plenum Press, New York, 1992), Chap. 2, pp. 21-107.

[5] A. J. Leggett et al., Rev. Mod. Phys. 59, 1 (1987).

[6] M. Büttiker and C. A. Stafford, Phys. Rev. Lett. 76, 495 (1996).

[7] A. O. Caldeira and A. J. Leggett, Phys. Rev. Lett. 46, 211 (1981).

[8] P. W. Anderson, G. Yuval, and D. R. Hamann, Phys. Rev. B 1, 4464 (1970).

[9] A. M. Tsvelick and P. G. Wiegmann, Adv. Phys. 32, 453 (1983); N. Andrei, K. Furuya, and J. H. Lowenstein, Rev. Mod. Phys. 55, 331 (1983).

[10] S. Chakravarty, Phys. Rev. Lett. 49, 681 (1982); A. J. Bray and M. A. Moore, ibid 49, 1545 (1982).

[11] V. V. Ponomarenko, Phys. Rev. B 48, 5265 (1993). 